\title{
Multi-gas spectroscopy using tailored mid-IR dispersive wave generated in $\mathrm{Si}_{3} \mathrm{~N}_{4}$ waveguide
}

\author{
Eirini Tagkoudi, ${ }^{1}$ Davide Grassani, ${ }^{2}$ Fan Yang, ${ }^{3}$ Camille-Sophie Brès ${ }^{1}$ \\ ${ }^{1}$ Ecole Polytechnique Fédérale de Lausanne (EPFL), Photonic Systems Laboratory (PHOSL), CH-1015, Switzerland \\ ${ }^{2}$ Dipartimento di Fisica, Università degli Studi di Pavia, Via Bassi 6, 27100 Pavia, Italy \\ ${ }^{3}$ Ecole Polytechnique Fédérale de Lausanne (EPFL), Group for fibre Optics (GFO), CH-1015, Switzerland \\ Authore-mail address: eirini.tagkoudi@epfl.ch
}

\begin{abstract}
We demonstrate simultaneous detection of acetylene, methane and ethane using milliwatt-level dispersive-wave generated in a $\mathrm{Si}_{3} \mathrm{~N}_{4}$ waveguide covering $2900 \mathrm{~cm}^{-1}-3380 \mathrm{~cm}^{-1}$ spectral region. This simple mid-IR absorption spectroscopy scheme achieves hundreds of ppm detection limit. (C) 2020 The Author(s)

OCIS codes: Nonlinear optics - Integrated optics (190.4390), Supercontinuum generation (320.6629), Absorption spectroscopy (300.1030).
\end{abstract}

\section{Introduction}

Soliton-induced dispersive wave (DW) emission in silicon nitride $\left(\mathrm{Si}_{3} \mathrm{~N}_{4}\right)$ waveguides is a way to efficiently transfer light from the near infrared (from a fiber laser) to the middle infrared (mid-IR). The combination of dispersion engineering of the waveguide and optimization of pumping wavelength while preserving the desired phase-matching conditions can significantly boost the efficiency of the light transfer, thus resulting in a powerful mid-IR emission from a simple configuration. As a matter of fact, the milliwatt power level in the mid-IR required for spectroscopy applications, can be obtained using only tens of $\mathrm{mW}$ of pump powers [1], overcoming previous limitations of power transfer in the spectral window between $3-4 \mu \mathrm{m}$ [2]. A proof-of-principle absorption spectroscopy experiment confirmed the value of such a DW-based source for detection of acetylene $\left(\mathrm{C}_{2} \mathrm{H}_{2}\right)[1]$. In this work, we experimentally demonstrate multiple gas linear absorption spectroscopy based on the mid-IR radiation generated on-chip using a tunable femtosecond fiber mode-locked laser (MLL) at 2.09 fiber $\mu \mathrm{m}$. We finely tune the DW center at $3.5 \mu \mathrm{m}$, as to cover the entire spectral window between $2900 \mathrm{~cm}^{-1}$ and $3380 \mathrm{~cm}^{-1}$, thus optimizing the performance of the device for 3 targeted gases. We leverage the efficient broadband nature of the DW for simple and direct absorption spectroscopy of acetylene $\left(\mathrm{C}_{2} \mathrm{H}_{2}\right)$, methane $\left(\mathrm{CH}_{4}\right)$ and ethane $\left(\mathrm{C}_{2} \mathrm{H}_{6}\right)$. Experimental results are compared with theoretical simulations of HITRAN database and a study of the signalto-noise (SNR) ratio was also performed for different integration times and coupled powers.

\section{Experimental setup and multi-gas detection}

The experimental setup is presented in Fig. 1(a). The pump is a commercial thulium-doped fiber MLL (Brevity $\lambda+$, NOVAE), with tunability from 2.07 to $2.10 \mu \mathrm{m}$. Light is coupled to the waveguide using a set of black diamond lenses. Upon propagation in the waveguide, DW emission occurs according to pre-designed phase matching conditions [1]. The output spectrum is then transmitted through a $5 \mathrm{~cm}$ gas cell filled with $\mathrm{C}_{2} \mathrm{H}_{2}, \mathrm{CH}_{4}$ and $\mathrm{C}_{2} \mathrm{H}_{6}$. After the interrogation of the 3-gas sample, output is collected with a collimator in a single-mode indium fluoride optical fiber and directed to an optical spectrum analyzer (OSA). We use a $2.15 \times 1.10 \mu \mathrm{m}^{2}$ waveguide for the multi-gas spectroscopy experiment. We can finely tune the DW position by shifting the pump wavelength in a specific waveguide (Fig.1(b)). The DW position can be therefore set as to provide the optimized performance given the targeted gas. For this specific experiment, we set the pump wavelength at $2.09 \mu \mathrm{m}$ resulting in a generated DW centered at $3.45 \mu \mathrm{m}$ offering the best overlap with $\mathrm{CH}_{4}$ and $\mathrm{C}_{2} \mathrm{H}_{6}$, while owing to its large bandwidth covering also the absorption lines of $\mathrm{C}_{2} \mathrm{H}_{2}$.

(a)

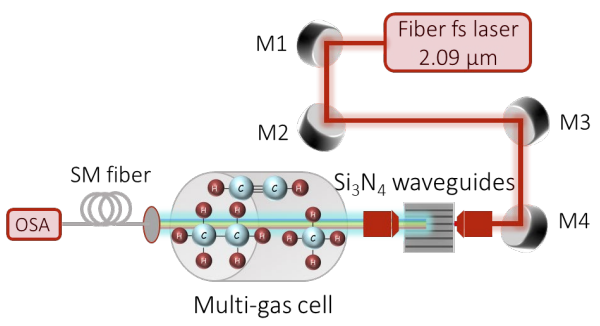

(b)

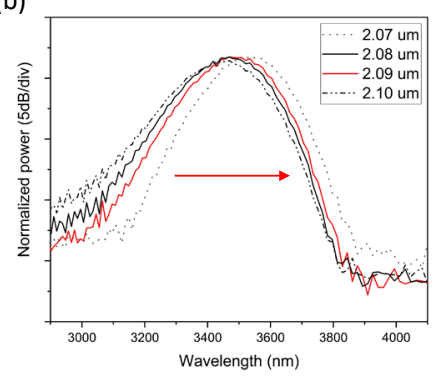

(c)

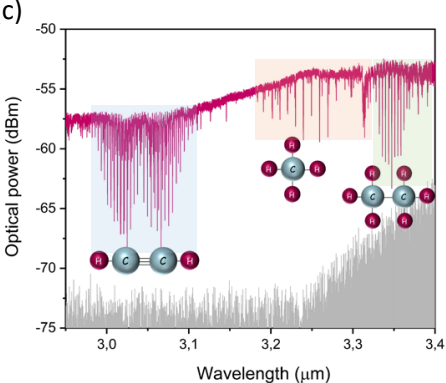

Fig.1(a) Experimental setup. M1-M4: mirrors, OSA: Optical spectrum analyzer (b) Fine tunability obtained through pump wavelength tuning, between 2.07 and $2.1 \mu \mathrm{m}$, for the dispersive wave position. (c) Parallel gas detection of $\mathrm{C}_{2} \mathrm{H}_{2}, \mathrm{CH}_{4}$ and $\mathrm{C}_{2} \mathrm{H}_{6}$ appears in the transmission spectrum at the output of the gas cell (red) observed on the OSA. Noise level is shown in grey color. 
The generated mid-IR DW covers a 3 to $3.8 \mu \mathrm{m}$ spectral range, however the spectroscopy was performed between 2.95 and $3.4 \mu \mathrm{m}$ due to the wavelength reach of the OSA. The overall transmission spectrum collected after the gas cell is presented in 1 (c). As clearly seen, the $\mathrm{C}_{2} \mathrm{H}_{2}$ absorption lines are in the range of $2.95 \mu \mathrm{m}-3.1 \mu \mathrm{m}, \mathrm{CH}_{4}$ main absorption lines lie in the $3.2-3.32 \mu \mathrm{m}$ range, while $\mathrm{C}_{2} \mathrm{H}_{6}$ lines appear between $3.32 \mu \mathrm{m}$ and $3.36 \mu \mathrm{m}$.

\section{Results and discussion}

The normalized experimental spectra we obtained for multi-gas spectroscopy using the Mid-IR DW and the comparison with the HITRAN model [3] are presented in Fig. 2 (a)-(c) for $\mathrm{C}_{2} \mathrm{H}_{2}, \mathrm{CH}_{4}$ and $\mathrm{C}_{2} \mathrm{H}_{6}$ respectively. The correspondence between the experimental data and the simulations based on HITRAN is clearly seen in the zoomin graphs shown in Fig. 2 (d)-(f), with the corresponding residuals plotted in black below. The standard deviation of the residual for the three gases using the spectral ranges shown in Fig. 2 (d)-(f) are calculated to be $\sigma=0.039$ for $\mathrm{C}_{2} \mathrm{H}_{2}, \sigma=0.036$ for $\mathrm{CH}_{4}$, and $\sigma=0.1$ for $\mathrm{C}_{2} \mathrm{H}_{6}$. The smallest standard deviation is calculated for methane, due to the higher spectral power of the mid-IR DW in the $\mathrm{CH}_{4}$ absorption lines neighborhood and to the relatively low noise level from the OSA in that area. On the other hand, the largest standard deviation for ethane comes from the higher noise level of the OSA at this wavelength range. Overall the analysis shows a very good agreement between the HITRAN model and the experimental measurements. The mole fractions of acetylene, methane and ethane are extracted from the fittings of the normalized absorbance spectra (Fig. 2 (a)-(c)). We obtain $\chi_{\mathrm{CH} 4}=5513 \mathrm{ppm}$ for $\mathrm{CH}_{4}, \chi_{\mathrm{C} 2 \mathrm{H} 2}=19760 \mathrm{ppm}$ for $\mathrm{C}_{2} \mathrm{H}_{2}$ and $\chi_{\mathrm{C} 2 \mathrm{H} 6}=9149 \mathrm{ppm}$ for $\mathrm{C}_{2} \mathrm{H}_{6}$. The noise-equivalent detection limit of our device, defined as $\chi / \mathrm{SNR}_{\max }$ is therefore $180 \mathrm{ppm}$ for $\mathrm{CH}_{4}, 782 \mathrm{ppm}$ for $\mathrm{C}_{2} \mathrm{H}_{2}$, and $977 \mathrm{ppm}$ for $\mathrm{C}_{2} \mathrm{H}_{6}$. The hundreds of ppm-level detection limit could be therefore anticipated with the use of a multi-pass gas cell. A SNR study as a function of the number of averaging times was also done in order to evaluate the performance of this device.

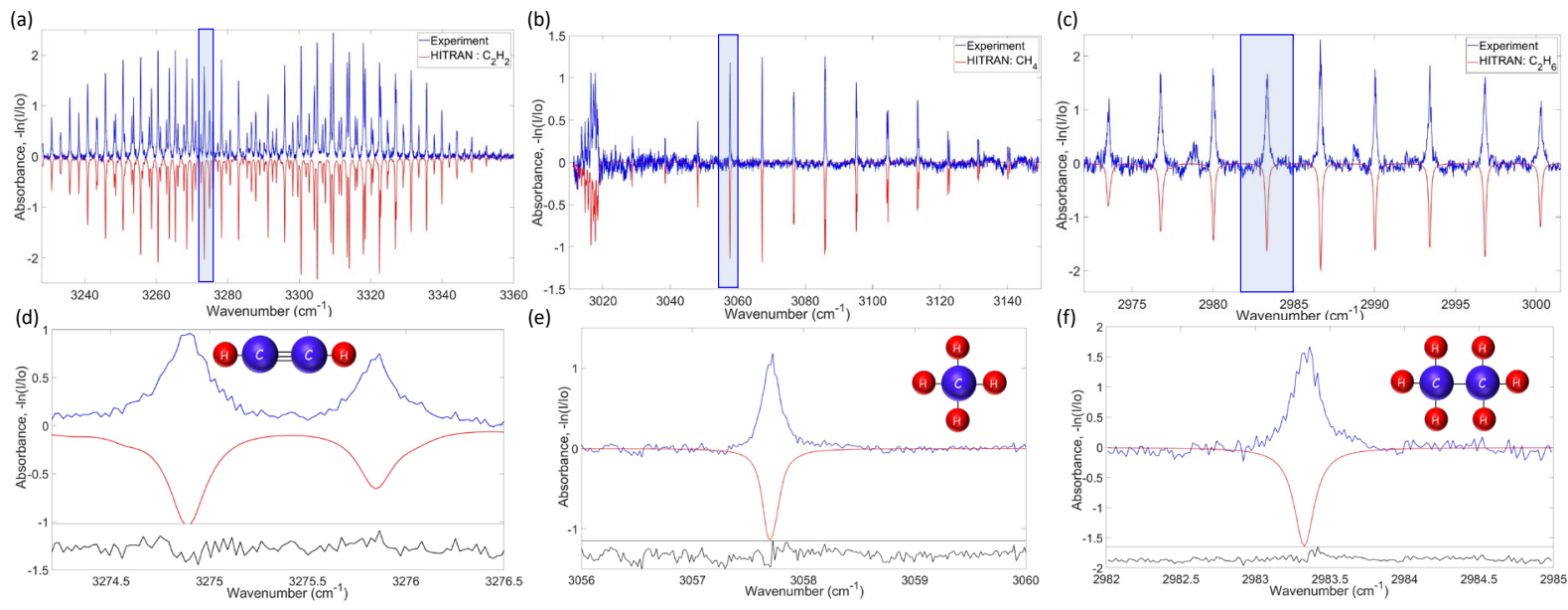

Fig.2 Normalized experimental gas absorbance (blue curve) and HITRAN database (red curve, inverted for clarity) for (a) acetylene, (b) methane and (c) ethane. The blue shaded areas show the area selected for the zoom in graphs shown in (d) for $\mathrm{C}_{2} \mathrm{H}_{2},(e) \mathrm{CH}_{4}$ and (f) $\mathrm{C}_{2} \mathrm{H}_{6}$. The residual (black line) is plotted on the same graph with an offset of (d) -1.25 , (e) -1.25 and (f) -1.80 .

In conclusion, we have experimentally demonstrated a compact, single-pass, multiple gas-phase species absorption spectroscopy device using a mid-infrared dispersive wave generated at $3.5 \mu \mathrm{m}$ in a dispersion tailored $\mathrm{Si}_{3} \mathrm{~N}_{4}$ nanophotonic waveguide utilizing $2 \mu \mathrm{m}$ fine-tuned fiber laser technology. We targeted the spectral region from $2.95 \mu \mathrm{m}$ to $3.5 \mu \mathrm{m}$, an important window for Mid-IR greenhouse gas spectroscopy and we achieved for the first time simultaneous detection of organic molecules such as acetylene, methane and ethane in one waveguidebased configuration. Experimental measurements are compared with HITRAN simulations and a signal-to-noise ratio (SNR) study is performed to explore the performance of the device.

Acknowledgements: This work was supported by the European Research Council under the grant agreement ERC-MATISSE and ERCPISSARRO. We would like to acknowledge K-lab of EPFL for the design and the fabrication of the chip used in the experiments.

[1] Grassani, D. et. al. 'Mid infrared gas spectroscopy using efficient fiber laser driven photonic chip-based supercontinuum', Nature Communications $10(1),(2019)$

[2] Guo, H. et. al. 'Mid-infrared frequency comb via coherent dispersive wave generation in silicon nitride nanophotonic waveguides', Nature Photonics, 12 (6), 330, (2018)

[3] Gordon, I.E. et. al. 'The HITRAN 2016 molecular spectroscopic database', Journal of Quantitative Spectroscopy and Radiative Transfer (2017) 